\title{
Anomalous origin of the right coronary artery from the pulmonary artery in association with congenital aneurysm of the sinus of Valsalva: angiographic diagnosis of a rare association
}

\author{
A Shyam Sundar, Keith A A Fox
}

\begin{abstract}
A 37 year old man presenting with acute heart failure, hypotension, and acute renal failure was diagnosed by cardiac catheterisation and angiography to have the rare combination of congenital aneurysm of the non-coronary sinus of Valsalva rupturing into the right ventricle, and an anomalous origin of the right coronary artery from the main pulmonary artery. The diagnosis could not be confirmed by transthoracic echocardiography in this patient. This combination of defects, confirmed at cardiac surgery, has not been reported before, and this case report highlights the importance of preoperative definition of congenital defects associated with an aneurysm of the sinus of Valsalva.
\end{abstract}

(Br Heart J 1992;68:330-2)

Anomalous origin of the right coronary artery from the main pulmonary artery is a rare congenital cardiac malformation. ${ }^{12}$ Twenty five cases with this condition have been reported to date, most as incidental findings, at necropsy, or during surgery; a few have been diagnosed by angiography. ${ }^{3}$ Congenital aneurysms of the sinus of Valsalva are comparatively rare $^{45}$ and may be associated with other anomalies including ventricular septal defect, aortic valve, and coronary artery anomalies. ${ }^{67}$ To the best of our knowledge, there have been no reported instances of anomalous origin of a right coronary artery from the pulmonary artery in association with congenital aneurysm of the sinus of Valsalva.

We report the preoperative diagnosis, by cardiac catheterisation and angiography, of the rare combination of ruptured aneurysm of the sinus of Valsalva and anomalous right coronary artery from the pulmonary artery in a 37 year old man presenting with acute heart failure, hypotension, and acute renal failure. The findings were confirmed at surgery.

\section{Case report}

A 37 year old man presented with a sudden illness. He had had a 48 hour history of palpitations followed by dyspnoea, recurrent vomiting, and anuria, and was admitted in acute renal failure and cardiogenic shock. He had previously felt entirely well and went swimming for 30 minutes, two days before admission to hospital. He had had no significant illness in the past and had no history of chest pain. On clinical examination, the patient was pale, cyanosed, tachypneic, and was hypotensive with wide pulse pressure and sinus tachycardia. He was in cardiac failure with raised jugular venous pressure, tender hepatomegaly, and bibasal chest crackles.

Cardiovascular examination showed cardiomegaly with a forcible cardiac impulse. There was a superficial continuous murmur (grade 3/6) heard at the lower left sternal border. The electrocardiogram showed sinus tachycardia without evidence of ventricular hypertrophy or myocardial ischaemia. The chest radiograph showed mild cardiac enlargement and pulmonary plethora. $\mathrm{He}$ was treated with ionotropes and haemofiltration followed by regular dialysis for control of his renal failure. $\mathrm{He}$ underwent transthoracic echocardiographic studies but high quality recordings could not be obtained because of his chest configuration.

Cardiac catheterisation showed raised right heart pressures with moderate pulmonary arterial hypertension $(50 / 25$ mean 35

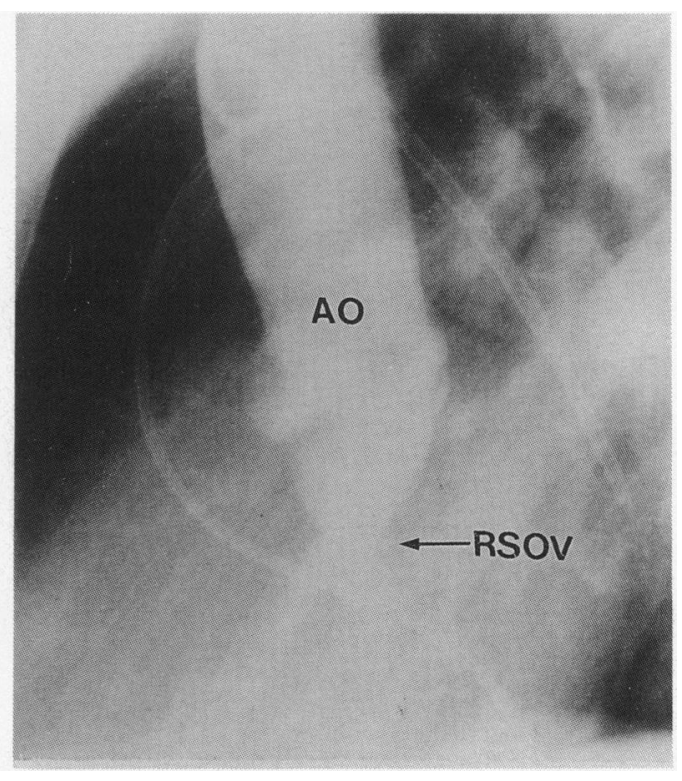

Figure 1 Aortogram (left lateral projection) showing ruptured aneurysm of the non-coronary sinus of Valsalva with a leak into the right ventricle. AO, aorta; $R S O V$, aneurysm of the ruptured sinus of Valsalva. 
Figure 2 Selective angiogram from main pulmonary artery showing anomalous pulmonary origin of the right coronary artery. $P A$, main pulmonary artery; $R C A$, right coronary artery.

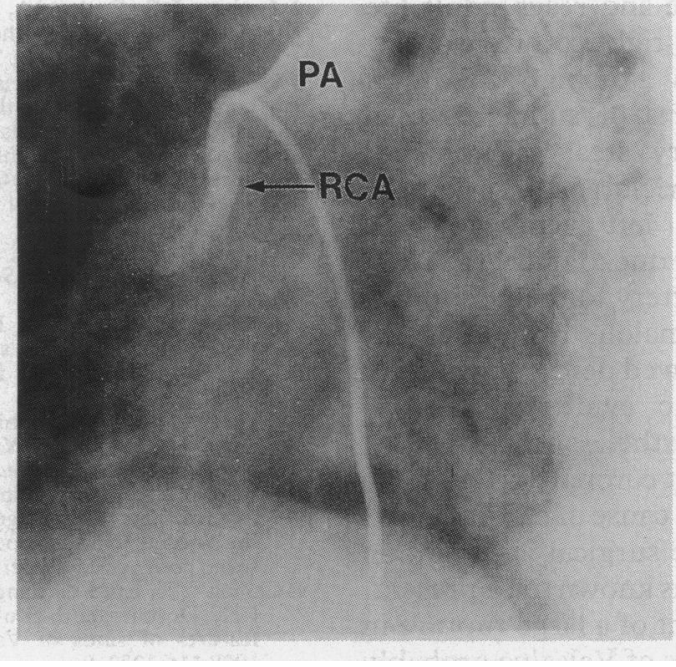

$\mathrm{mm} \mathrm{Hg}$ ). There was a step up in oxygen saturation in the right ventricle with a left to right shunt of $4: 1$. Aortic root angiography showed an aneurysm of the non-coronary sinus of Valsalva rupturing into the right ventricle (fig 1). Coronary angiography showed a normal left coronary artery with collaterals to the right coronary artery. The large right coronary artery filled retrogradely from the left system and showed flow of contrast material into the main pulmonary artery from which it originated. Anomalous pulmonary origin of the right coronary artery was documented by selective cineangiography from the main pulmonary artery (fig 2). The patient also had a patent foramen ovale and a left superior vena cava draining into the coronary sinus.

The patient underwent successful operation and at surgery, the findings of ruptured aneurysm of the non-coronary sinus of Valsalva opening into the right ventricle and anomalous origin of the right coronary artery from the main pulmonary artery were confirmed. The aneurysm of the sinus of Valsalva produced the previously described "windsock" deformity and was excised, leaving a slit-like defect between the aortic wall and the aortic annulus. This was closed with sutures. The anomalous right coronary artery could not be seen on external inspection but subsequent dissection and exploration showed it to arise from the right side of the pulmonary artery and it was reimplanted into the ascending aorta. No heart murmurs were heard in the postoperative period. Renal function gradually recovered to normal and a repeat Swan Ganz catheterisation six weeks after operation showed normal pressures and oxygen saturation. The patient is currently symptom free 18 months after operation.

\section{Discussion}

Aneurysms of the sinus of Valsalva are rare, accounting for $0.43 \%$ and $0.14 \%$ of open heart operations done at two major cardiac surgical centres. ${ }^{45}$ They are frequently associated with other congenital cardiac defects such as ven- tricular septal defect and aortic valve anomalies. ${ }^{6}$ Anomalies of the coronary artery may complicate the surgical repair especially when only discovered at operation for rupture of the aneurysm of the sinus of Valsalva. For example, a single coronary artery was discovered at operation when coronary angiography was not performed.

Although aneurysms of the sinus of Valsalva may be detected by echocardiography, in subjects in whom high quality recordings are obtainable, this case report shows the clinical importance of defining any associated congenital anomalies. Unless such concomitant lesions can be defined by echocardiography, it may be necessary to undertake angiography in patients with ruptured aneurysm of the sinus of Valsalva.

Echocardiography is a useful non-invasive tool in diagnosing aneurysms of the sinus of Valsalva. ${ }^{8}$ Cross sectional and Doppler echocardiography, however, even in experienced hands, have missed such aneurysms, which were subsequently diagnosed by cardiac catheterisation and confirmed at surgery. ${ }^{9}$ Despite a clinical diagnosis before echocardiography of a ruptured aneurysm of the sinus of Valsalva, the transthoracic echocardiographic recordings were inadequate to confirm the diagnosis in this compromised and poorly echogenic subject. Transoesophageal echocardiography, with Doppler colour flow mapping, would be the investigation of choice for such suspected ruptures, particularly where transthoracic recordings are inadequate. ${ }^{10}$ This would not, however, have shown the associated anomaly of the coronary artery. Transoesophageal echocardiography was not available to us when this patient presented.

The definitive diagnosis of ruptured aneurysm of the sinus of Valsalva can be established by cardiac catheterisation and angiography, as can associated cardiac defects detected, including anomalies of the coronary artery.

Anomalous origin of the right coronary artery from the main pulmonary artery is extremely rare with a reported incidence of $0.002 \%$ in a series of 126595 patients undergoing coronary angiography over a 28 year period at the Cleveland Clinic Foundation. ${ }^{11}$ Twenty five reported cases are available for review. ${ }^{12-14}$ Isolated cases of patients with associated congenital heart disease, including atrial septal defect, tetralogy of Fallot with patent ductus arteriosus, pulmonary atresia with intact ventricular septum, and aortopulmonary window with and without ventricular septal defect have been reported..$^{231314}$ An anomalous origin of the right coronary artery from the pulmonary artery, as far as we know, has not been previously described in association with congenital aneurysm of the sinus of Valsalva.

By contrast with patients who have the far more common condition of anomalous pulmonary origin of the left coronary artery and who often present in infancy with ischaemia and congestive heart failure, ${ }^{3}$ most patients with anomalous origin of the right coronary 
artery are symptom free and reach adult life. Anomalous origin of the right coronary artery, however, is not always a benign condition. There have been reports of sudden cardiac death $^{215}$ and a coronary steal phenomenon whereby the right ventricle and inferior and septal regions of the left ventricle were deprived of blood supply due to retrograde flow from the left coronary artery to the pulmonary artery through the anomalous right coronary artery. ${ }^{16}$ Our patient showed neither symptoms nor electrocardiographic evidence of myocardial ischaemia. Nevertheless, the fact that anomalous origin of right coronary artery from the pulmonary artery is a cause of cardiac arrest may justify prophylactic surgical intervention if this coronary anomaly is known to be present.

The acute development of a large rupture in the aneurysm of the sinus of Valsalva probably accounted for the dramatic presentation in our patient. As a result of the anomalous large right coronary artery, however, ischaemia of right ventricle and posteroinferior regions of the left ventricle could have contributed to the development of heart failure.

Our patient highlights the usefulness of cardiac catheterisation and angiography in the accurate preoperative delineation of this rare combination of lesions that were confirmed at surgery.

We gratefully acknowledge the surgical expertise of Mr E G Butchart and Mr A J Bryan in the management of this patient.

1 Roberts WC. Major anomalies of coronary arterial origin seen in adulthood. Am Heart $J$ 1986;111:941-63.
2 Lerberg DB, Ogden JA, Zuberbuhler JR, Bahnson HT. Anomalous origin of the right coronary artery from the pulmonary artery. Ann Thorac Surg 1979;27:87-94.

3 Neufeld HN, Schneeweiss A. Anomalous origin of the coronary arteries from the pulmonary artery. In: Neufeld HN, Schneeweiss A, eds. Coronary artery disease in infants and children. Philadelphia: Lea and Febiger, 1983:1-30.

4 and children. Philadelphia: Lea and Febiger, 1983:1-30. Meyer J, WuKasch DC, Hallman GL, Cooley DA.
Aneurysm and fistula of the sinus of Valsalva. Ann Thorac

5 Henze A, Huttunen H, Bjork VO. Ruptured sinus of Valsalva aneurysms. Scan $J$ Thorac Cardiovasc Surg 1983;17:249-53.

6 Nowicki ER, Aberdeen E, Friedman S, Rashkind WJ Congenital left aortic sinus-left ventricular fistula and review of aortocardiac fistulas. Ann Thorac Surg 1977, 23:378-88.

7 Chamsi-Pasha H, Gandhi RG, Logan WFW. Ruptured aneurysm of sinus of Valsalva co-existing with a single coronary artery. Eur Heart $J$ 1989;10:180-1.

8 Sahasakul Y, Panchavinnin P, Chaithiraphan S, Sakiyalak P. Echocardiographic diagnosis of a ruptured aneurysm of the sinus of Valsalva: operation without catheterisation in seven patients. Br Heart J 1990;64:195-8.

9 Chiang CW, Lin FC, Fang BR, Kuo CT, Lees YS, Chang CH. Doppler and two-dimensional echocardiographic features of sinus of Valsalva aneurysm. Am Heart 1988;116:1283-8.

10 Abdelkhirane C, Roudaut R, Dallocchio M. Diagnosis of ruptured sinus of Valsalva aneurysms: Potential value of transesophageal echocardiography. Echocardiography 1990;7:555-60.

11 Yamanaka O, Hobbs RE. Coronary artery anomalies in 126595 patients undergoing coronary arteriography. Cathet Cardiovasc Diagn 1990;21:28-40

12 Mahdyoon H, Brymer JF, Alam M, Khaja F. Anomalous right coronary artery from the pulmonary artery presentright coronesentAm Heart $J 1989 ; 118: 182-4$.

13 Brouwer MHJ, Beaufort-Krol GCM, Talsma MD. Aortopulmonary window associated with an anomalous origin of pulmonary window associated with an anomalous origin

14 Gerlis LM, Ho SY, Milo S. Three anomalies of the coronary arteries co-existing in a case of pulmonary atresia with intact ventricular septum. Int $J$ Cardiol 1990;29:93-5.

15 Wald S, Stonecipher K, Baldwin BJ, Nulter DO Anomalous origin of the right coronary artery from the pulmonary artery. Am J Cardiol 1971;27:677-81.

16 Mintz GS, Iskandarian AS, Bernis CE, Mundth ED, Owen JS. Myocardial ischemia in anomalous origin of the righ coronary artery from the pulmonary trunk. Am J Cardio 1983;51:610-2. 